\title{
Analysis of cylindrical roller bearings design in order to optimize the classical process using FEM
}

\author{
Alin Marian Pușcaşu ${ }^{*}$, Octavian Lupescu, and Ana Bădănac \\ Technical University "Gheorghe Asachi” of Iasi, Department of Machine Manufacturing and \\ Industrial Management, B-dul Mangeron, 59A, Iaşi, 700050, România
}

\begin{abstract}
This paper presents the structural and functional improvement of cylindrical roller bearings using finite element method. This method has a widely application and enjoys by an extended use in structural areas thermal analysis, mechanical and vibration. The advantages of the finite element method (FEM-Finite Element Method) are numerous and important. In case of conception a design it can be studied and modelled the structure behaviour in various media load in real time, therefore, based on the obtained results, the model can be modify before creating the final drawings for execution. Once is developed the CAD model, using FEM method it can be analyse the design structure in detail. Therefore using FEM can save time and money by reducing the required number of prototypes.
\end{abstract}

\section{Introduction}

As it is known, the bearings are essentials part of all rotating machines whose function is to support the machines. More than $90 \%$ of use rolling element bearings and their failure may lead to a catastrophic failure of the machine. Radial bearings are considered as the most critical components in industrial applications, [1]. Given the fact that the bearings are designed to withstand high loads and are mounted on complex and expensive cars and machinery, nothing can be left to chance in the bearing selection process suitable for the application in question [2]. Strictly related to design technologies, developed concern for research and development relating to the materials used, the heat treatment of surfaces, machining, surveying, contacts, profiles, analysis and simulation software's, leading to high levels of knowledge crucial to continuously improve technological conditions incorporated in the final product. The bearing types currently used in practice are: cylindrical roller bearings, thrust bearings, tapered roller bearings and spherical bearings [3]. In the case of cylindrical roller bearings they are manufactured in a wide variety of construction types and sizes, especially on single row rollers, and in some situations two or more rows of rollers, with a roll cages or side by side rollers. In the cylindrical roller bearings, the rollers are guided by the side of one of the rings collars.

* Corresponding author: puscasu_alin@yahoo.com 
In the case of bearing with cage, ring collar and cage with retained rollers can be extracted from the other ring; this means that the bearings are removable. With this, mounting and dismounting of bearings becomes much easier, especially in places where due to loading conditions, tight fits are required for both rings.

\section{Method}

To optimize the design cylindrical roller bearings was done first resistance analysis benchmarks. Analysis was prepared using the finite element method. This method consists of a continuous body mesh and finished in several finite element (FEM - Finite element method). The mesh structure is meant in subdivision mathematical model with simple geometric form, which does not overlap, called finite elements. If the answer to each finite element simulations is expressed on a finite number of degrees of freedom that represent the values of unknown function (movement function) in a number of crucial points. Thus the answer to the mathematical model will result as an approximation of the response obtained by assembling discrete model answers all model elements. This method has wide application and enjoys widespread use in areas of structural, thermal analysis, mechanical and vibration. If we can conceive a design study model and structure behaviour in various media load in real time, based on the results, we may change the model before creating the final drawings. Once the CAD model is developed, using FEM method we can analyse in detail a structure. FEM programs, in our case ANSYS software found at S.C. Rulmenti S.A. Barlad has a library of tens of types of finite elements, from which the user must choose the most effective type for bearing structure. According to [4] ANSYS simulation software allows the designer to predict with confidence how the product will work under real conditions. Simulation is a powerful modular system. ANSYS Mechanical is a complete analysis FEM for structural type analysis, including linear and nonlinear dynamic studies.

The product offers a complete set of elements, material models and equation solving a wide range of mechanical design problems. FEM contains no "indications " or restrictions in this regard. However to ensure efficient model requires that the types of finite elements are chosen depending on numerous factors and conditions, the first of which is functionality, distribution of loads and tensions, to assure the DOF and limit certain others. By selecting ANSYS Mechanical simulation as a tool, S.C. Rulmenti S.A. Barlad has a large gain in terms of high computing power and response pattern of its products. It covers a wide range of properties, such as tension, strain, vibration characteristics, thermal characteristics, and durability.

\section{Analyse and Results}

For the achievement of experimental research was used a cylindrical roller bearing type NJ with brass cage. Components for this type of bearing is used in CHM speed reducer shaft, was first designed in Creo Parametric software, (Figure 1). Then the geometry for finite element analysis was exported Ansys 17.0, (Figure 2). After importing the geometry, we have conducted a series of optimizations on the components of the geometry in order to simplify and to reduce the possibility of calculation errors.

As a first result of importation, the bearing surfaces are cut symmetrically, so the first stage is to use MERGE command and merge the surfaces (Figure 3). In the second phase were carried out a number of sections for each element of the ensemble, using commands such as; slice, delete, flat, extruded, the result being represented by a set cut in as simple bodies, in order to simplify our analysis (Figure 4). In the third step was placed in a shaft assembly located on the inner ring (Figure 5). 

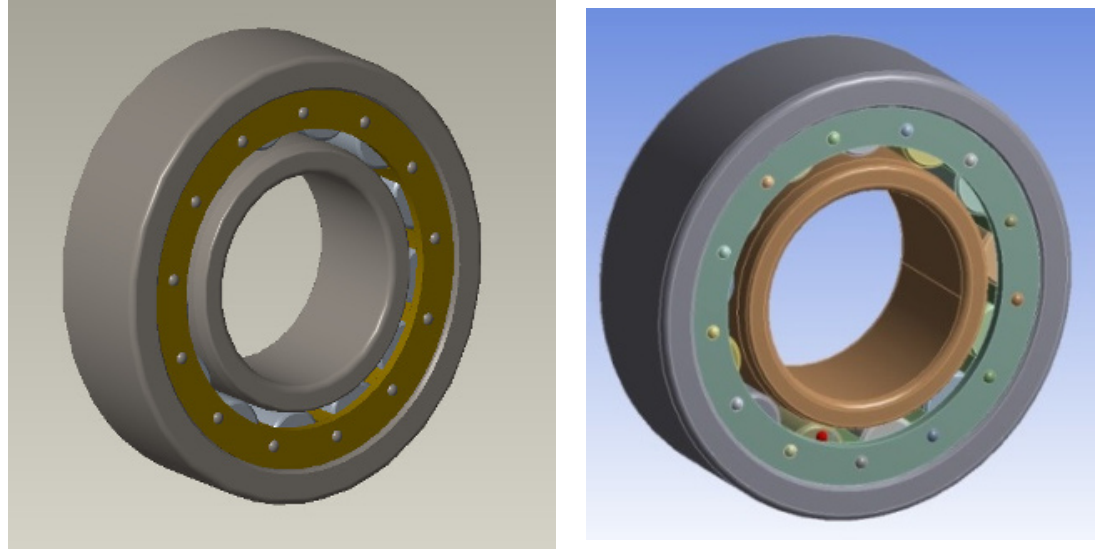

Fig.1. Geometry in CREO 3.0.

Fig.2. Geometry in ANSYS 17.0.

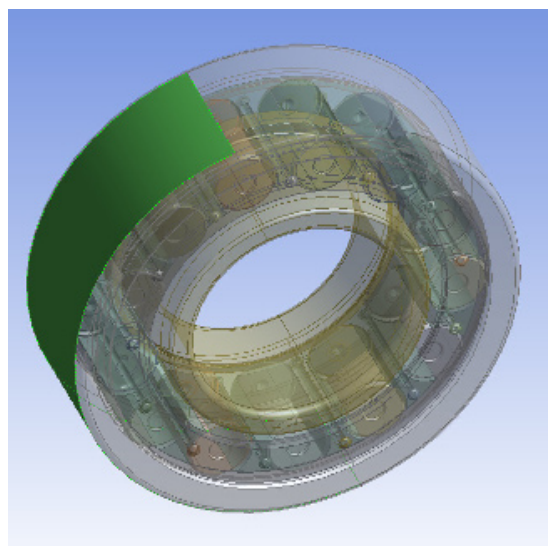

Fig.3. Merging separated faces.

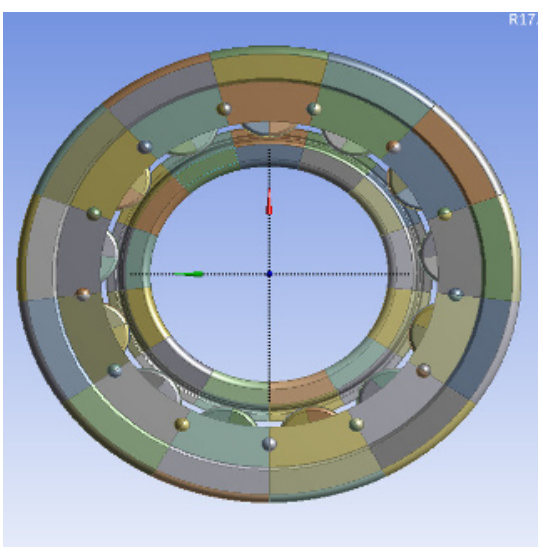

Fig.4. Dividing bodies.

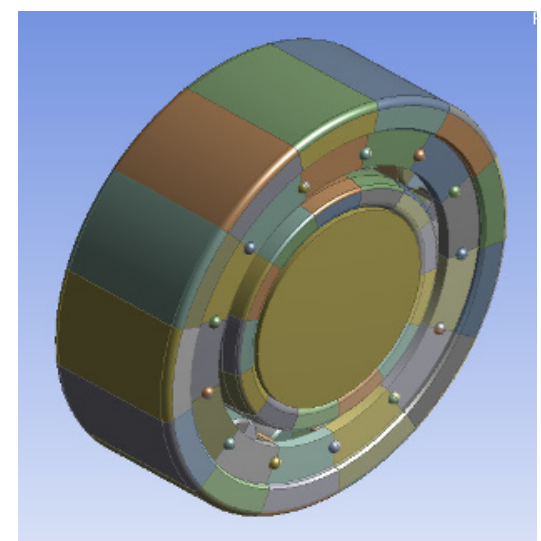

Fig.5. Shaft on the inner ring. 
After completing the operations to simplify geometry, we passed to the next stage, namely the introduction of mechanical model, at this stage were established the contacts between parts, couplings, meshing (Figure 6).

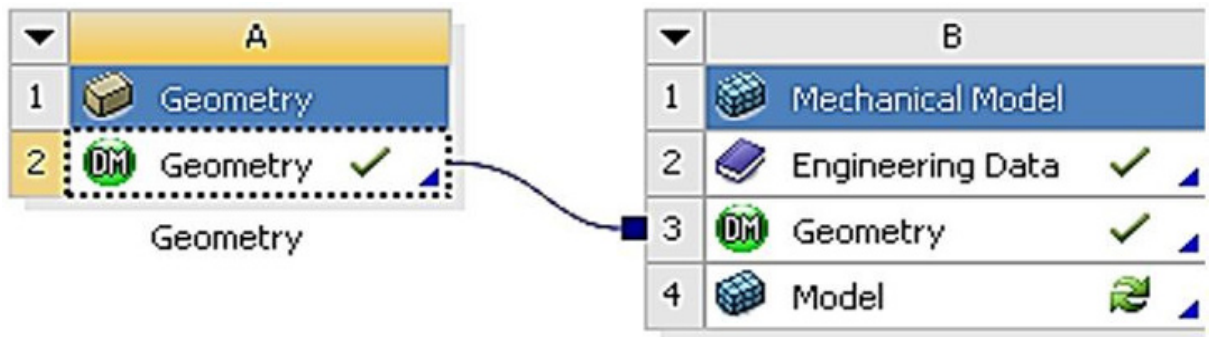

Fig.6. Mechanical Model.

It has achieved a uniform bearing mesh, with a number of nodes and elements as small as possible, to ease the process of calculating and also to shorten the settlement period. Optimization of the mesh was carried out in order to obtain a continuous mesh and a correlation between the number of finite elements and the number of associated nodes, Figure 7. I chose a simple type generally fixed mesh, a mesh type Hex roller type and a mesh cage and tetrahedrons for rivets. It chose a maximum size of items of $2 \mathrm{~mm}$ and a minimum allowable size of $0.5 \mathrm{~mm}$ with a growth rate of $1.2 \mathrm{~mm}$. As shown in Figure 8 , the resulting mesh is top quality with a relatively small number of nodes respectively elements in view of the structure and complexity. Also for rings was established a custom mesh.

For this present study it was chosen a MultiZone mesh with features recommended sizing, Hexa with each element of $2 \mathrm{~mm}$ mesh, free method of uniform surface type and concentration of each element type nodes Kept. For the rollers I selected a method hex dominant node with a concentration on each element type dropped. For cage and cage cover was admitted a mesh patch type and method conforming tetrahedrons.

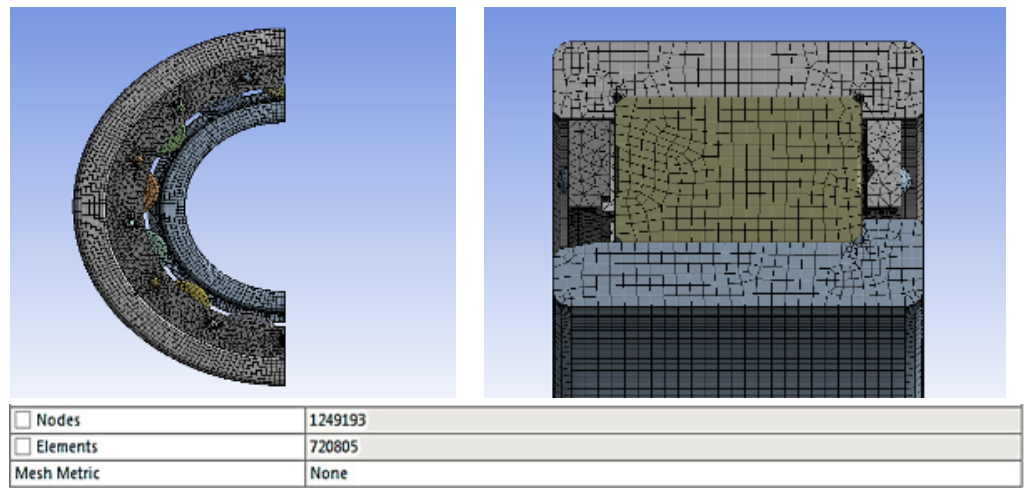

Fig.7. Mesh 1.

If was chosen the rivet, as a method hex dominant surface mesh with a quad / tri. This is given the low number of nodes and elements and how uniform meshing for analysis was chosen the first method of meshing. At the end of the round mesh was introduced a condition that would be provided contacting as many knots possible between rollers and rings, this command is going node. 

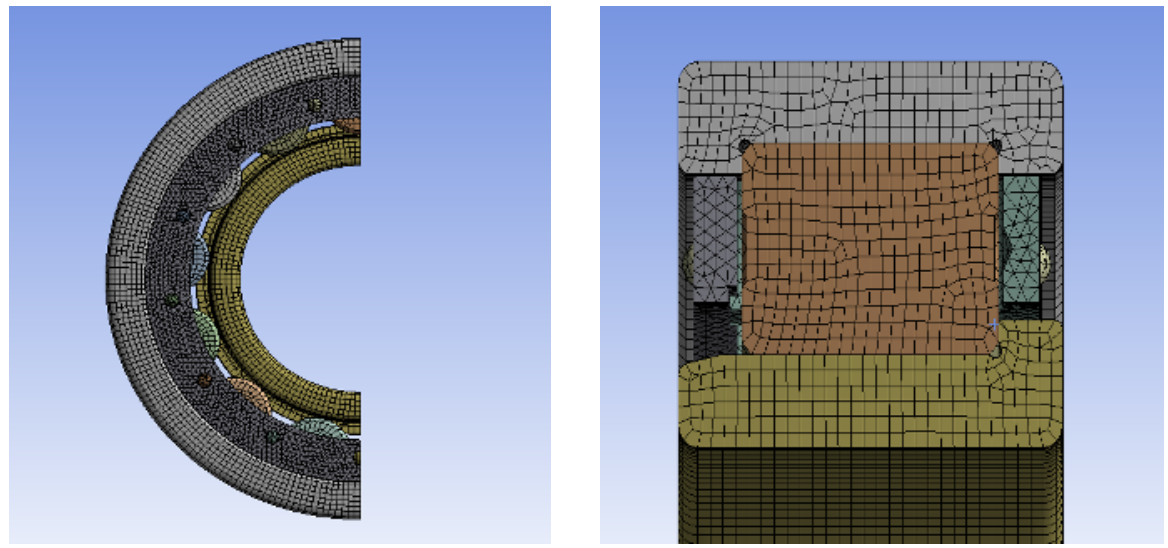

\begin{tabular}{l|l} 
Statistics \\
\hline$\square$ Nodes & 865133 \\
\hline$\square$ Elements & 447160 \\
\hline Mesh Metric & None \\
\hline
\end{tabular}

Fig.8. Mesh 2.

At this stage, were established limits to which the bearing is subjected, the moving part, the fixed part and axial displacements. The fixed part of the bearing is outer ring, was imposed displacements constrains and rotations in all 3 axes (fig.9).

A load was applied on the inner ring of the bearing, radial load limit request the catalogue is $376 \mathrm{Kn}$, but for the analysis was elected a calculated value of $80 \mathrm{Kn}$. The charge is applied on the inner ring through the shaft, (fig.10).

In the Figure 11 are presented the results of static analysis for contact pressure and respective in Figure 12 for bearing life.

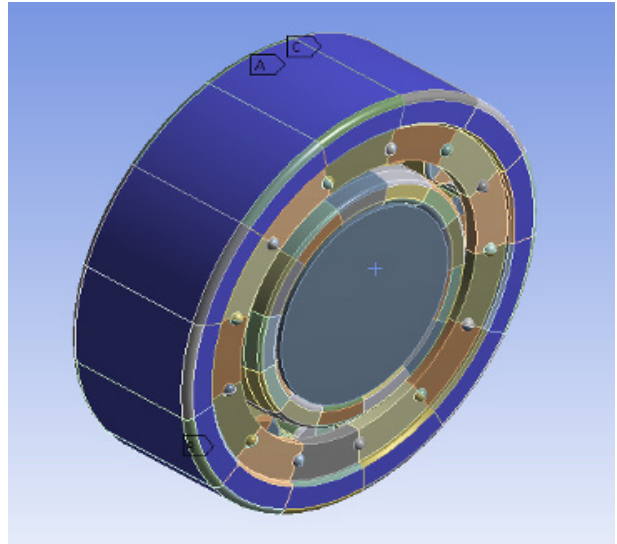

Fig.9. Outer ring fixed.

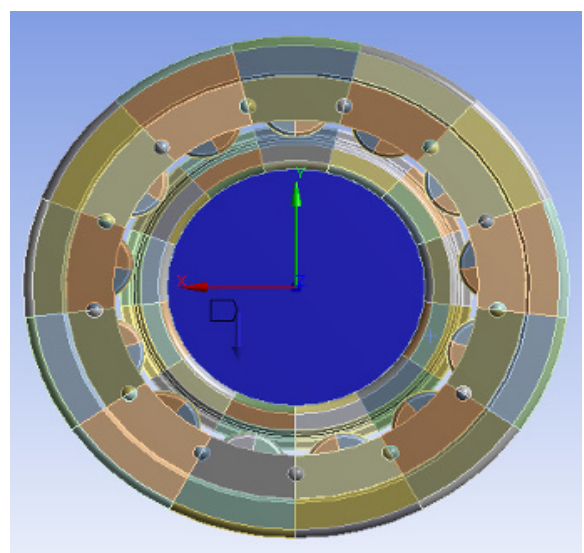

Fig.10. Shaft loaded. 


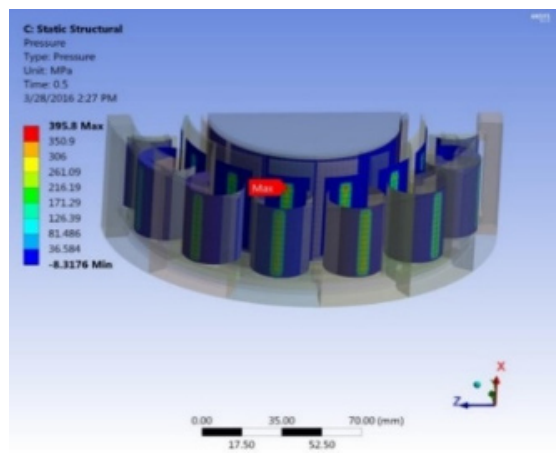

a) Element contact

Fig.11. Contact pressure.

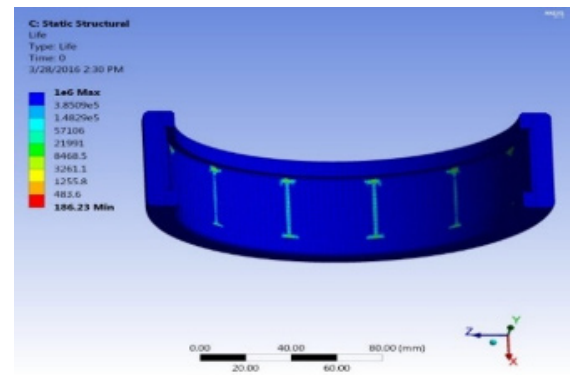

a) The life time of the outer ring

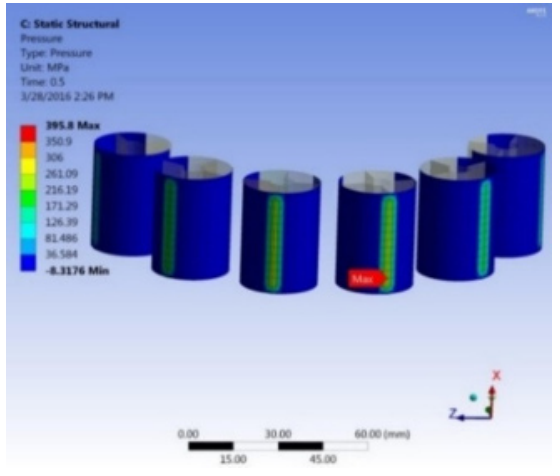

b) Contact surface of the rollers

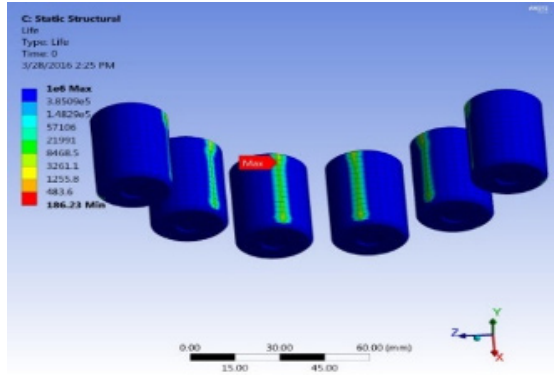

b) The life time of the rollers

Fig.12. Bearing life.

\section{Conclusion}

Analysis of the results led to the following conclusion: finite element analysis software Ansys allowed through static structural analysis the accumulation of information's about stress and deformation of the structure rollers and rings, the life of the bearing was improved and load distribution on the rollers changed disappeared from the discharges end of the rollers. They reveal spikes on the edges of the rollers. The value of the bearing life is similar to the amount recorded in the S.C. Rulmenti S.A. Barlad catalogue.

\section{References}

1. D. R. Pradhan, Mohan Handbook of Research on Manufacturing Process Modelling and Optimization Strategies, (2014)

2. Y. Zhang, T. Qingchang, Z. Kuo1, L. Jiangang, Analysis of Stress and Strain of the Rolling Bearing by FEM method, international conference on applied physics and industrial engineering. Physics procedia 24, pp. 19-24, (2012).

3. P. Chunjun, Thesis of Static Analysis of Rolling Bearings Using Finite Element Method, (2009).

4. E. Suli, Lecture Notes on Finite Element Methods for Partial Differential Equations, Mathematical Institute University of Oxford, (2012). 